\title{
ENEA-Bologna production and testing of JEFF-3.1 multi-group cross section libraries for nuclear fission applications
}

\author{
Massimo Pescarini ${ }^{1, a}$, Valentin Sinitsa ${ }^{2, b}$, and Roberto Orsi ${ }^{1}$ \\ ENEA, Ente per le Nuove Tecnologie, l'Energia e l'Ambiente, Centro Ricerche "E. Clementel” Bologna, 40129 Bologna, Italy \\ 2 ENEA consultant, formerly at SSC RF IPPE, State Scientific Center of the Russian Federation, \\ Institute for Physics and Power Engineering, Obninsk, Russian Federation
}

\begin{abstract}
The ENEA-Bologna Nuclear Data Group produced the JEFF-3.1 VITJEFF31.BOLIB and MATJEFF31. BOLIB fine-group coupled neutron and photon $(199 n+42 \gamma)$ cross section libraries for nuclear fission applications, respectively in AMPX and MATXS format, with the same specifications and energy group structure of the ENDF/BVI.3 VITAMIN-B6 American library. Each library, containing 181 nuclide cross section files, was generated from the same set of cross section data files in GENDF format, obtained through the Bondarenko (f-factor) method, with an ENEA-Bologna revised version of the GROUPR module of the NJOY-99.160 system. Collapsed working libraries of self-shielded cross sections in FIDO-ANISN format, used by the deterministic transport codes of the DANTSYS and DOORS systems, can be generated from VITJEFF31.BOLIB and MATJEFF31.BOLIB through, respectively, further data processing with an ENEA-Bologna revised version of the SCAMPI system and with the TRANSX code. This paper describes the methodology and specifications of the data processing performed and presents some results of the VITJEFF31.BOLIB validation.
\end{abstract}

\section{Introduction}

In recent years, the three-dimensional (3D) deterministic discrete ordinates (SN) transport codes using group-wise cross section libraries extended their practical simulation capability to an enlarged variety of nuclear systems with different neutron spectra and high geometrical complexity. In particular they increased their competitiveness with respect to the 3D Monte Carlo codes: comparable or even more convenient performances in terms of CPU times were in fact obtained with the same calculation precision, similar description capability of complex geometries and suitable simulation of different neutron and photon spectral conditions. Various factors concurred to determine specifically the increased flexibility and convenience of the 3D SN codes: the impressive increased performance of modern computers, innovative fine-group cross section libraries with up-scatter cross sections like VITAMIN-B6 [1], VITJEF22.BOLIB [2] or MATJEF22.BOLIB [3] and new ancillary pre-postprocessor systems of programs as BOT3P [4], dedicated to simplify and to strengthen the preparation and the graphical verification of the input of $2 \mathrm{D}$ and $3 \mathrm{D}$ geometrical models. Up to recent times, only the 3D Monte Carlo codes could obtain satisfactory results with high calculation precision in the description of complex geometries while at present, with the recent availability of new pre-post-processor systems, the $3 \mathrm{D}$ $\mathrm{SN}$ codes increased dramatically their geometrical simulation capability and this induced an increased interest to give more availability of updated group-wise cross section libraries. This is the background which encouraged the ENEA-Bologna

\footnotetext{
${ }^{a}$ Presenting author,

e-mail: massimo.pescarini@bologna.enea.it

$b$ Present address: ENEA, Centro Ricerche "E. Clementel" Bologna, 40129 Bologna, Italy
}

Nuclear Data Group to continue the generation of fine-group cross section libraries of VITAMIN-B6-type and derived collapsed working libraries together with a further development of BOT3P. The generation of the VITJEFF31.BOLIB (AMPX format) and MATJEFF31.BOLIB (MATXS format) fine-group coupled neutron and photon $(199 n+42 \gamma)$ cross section libraries for nuclear fission applications, based on the JEFF-3.1 European nuclear data file [5], is described in this paper. It is underlined that this work was performed under contract with OECD/NEA Data Bank (OECD/NEADB).

\section{Processing codes and procedures}

The VITJEFF31.BOLIB/MATJEFF31.BOLIB libraries, generated through the NJOY-99.160 data processing system [6], were derived from the same set of cross section files in GENDF format. The following modules of NJOY were used to process neutron interaction (n-n), gamma ray production (n- $\gamma$ ) and gamma ray interaction $(\gamma-\gamma)$ data, from the JEFF-3.1 incident neutron and photo-atomic data in ENDF-6 format into the GENDF format. Specifically, the MODER, RECONR, BROADR, THERMR, HEATR, GASPR, PURR, GROUPR modules were used for the incident neutron data while the MODER, RECONR and GAMINR modules were used for the photo-atomic data. A correction patch for GROUPR (ENEA-Bologna revised version [7]) was prepared to extend the group-wise data processing capability to evaluated data files including non Cartesian interpolation schemes as, e.g., 69 JEFF-3.1 evaluated files which could not be processed correctly through NJOY-99.160 or through all previous versions officially released. These evaluated files contain, in particular, secondary neutron energy distributions $(\mathrm{MF}=5)$, presented as an arbitrary tabulated function $(\mathrm{LF}=1)$ with the unit base interpolation law INT $=22$. Up to NJOY-99.160, 
GROUPR could not process them correctly because the GETSED subroutine could not deal with secondary neutron energy distributions involving the non Cartesian interpolation schemes INT $=11-15$ and INT $=21-25$. It is underlined that the correction patch officially introduced for the first time in GROUPR of NJOY-99.161 can only treat the INT $=21-25$ non Cartesian interpolation schemes, differently from the ENEA revised version of GROUPR which can treat all the INT interpolation schemes previously cited and was requested, tested and freely distributed for the first time by OECD/NEADB with NJOY-99.112. Then an ENEA-Bologna revised version of the SMILER module [7] of the SCAMPI data processing system [8] was used to translate the multi-group data from the GENDF format into the AMPX master library format of VITJEFF31.BOLIB. It is underlined that, in particular, this ENEA revised version of SMILER permits to obtain separately the prompt fission spectrum $(\mathrm{MF}=6$ and $\mathrm{MT}=18)$, the delayed fission spectrum $(\mathrm{MF}=5$ and $\mathrm{MT}=455)$ or the total fission spectrum needed for fixed source transport calculations, differently from the original ORNL SMILER version, used to generate VITAMIN-B6 and VITJEF22.BOLIB, which can permit to obtain only the prompt component. Then the module AIM of SCAMPI was used to convert the master cross section library from binary to BCD format. Concerning MATJEFF31.BOLIB, the MATXSR module of NJOY was used to translate the group-wise data from the GENDF format into the MATXS BCD cross section interface format. The availability of VITJEFF31.BOLIB and MATJEFF31.BOLIB permits now to collapse new self-shielded broad-group working cross section libraries which, e.g., can be produced in the FIDO-ANISN format only with the ENEA revised version of SCAMPI from VITJEFF31.BOLIB and with the TRANSX [9] code from MATJEFF31.BOLIB. More specifically, the cross sections in AMPX format can be collapsed only through an ENEA-Bologna revised version of the MALOCS module [7], can be self-shielded through the BONAMI module and finally can be produced into the AMPX or FIDO-ANISN formats. The cross sections in AMPX format can be used by the XSDRNPM code (1D SN), included in the AMPX/SCAMPI systems. Moreover the cross sections in FIDO-ANISN format can be used by the SN codes ONEDANT (1D), TWODANT (2D) and THREEDANT (3D) of the DANTSYS system [10], by the ANISN-ORNL (1D), DORT (2D) and TORT (3D) similar codes of the DOORS system [11] and, finally, by the 3D Monte Carlo code MORSE. The present calculations were performed on a Personal Computer (CPU INTEL Pentium III, $448 \mathrm{MB}$ of RAM; FSF-g77 version 0.5.26 FORTRAN compiler for NJOY and f77 Absoft version 5.0 FORTRAN 77 compiler for SCAMPI) with the Linux Red Hat 7.1 operative system.

\section{Library specifications}

A set of 181 JEFF-3.1 cross section files was processed for the VITJEFF31.BOLIB/MATJEFF31.BOLIB libraries. The increased number of materials with respect to the VITJEF22.BOLIB/MATJEF22.BOLIB (138 materials) and VITAMIN-B6 (120 materials) libraries is caused by the progressive availability of evaluated isotopic data files in substitution of natural element files. The Bondarenko (f-factor) method [12] was used for handling resonance selfshielding and temperature effects. As for VITAMIN-B6, all the 175 standard (not bound) nuclides were processed at 4 temperatures $\left(300^{\circ} \mathrm{K}, 600^{\circ} \mathrm{K}, 1000^{\circ} \mathrm{K}\right.$ and $\left.2100^{\circ} \mathrm{K}\right)$ and most materials were processed with 6 to 8 values for the background cross section $\sigma_{0}$. Nearly all materials were processed with the following values of $\sigma_{0}: 1,10,1.0 \mathrm{E}+2,1.0 \mathrm{E}+3$, $1.0 \mathrm{E}+4$ and $1.0 \mathrm{E}+10$ barns. Each nuclide was processed at the same corresponding $\sigma_{0}$ values adopted in the production of VITAMIN-B6. For consistency with most other libraries, infinitely dilute $\sigma_{0}(1.0 \mathrm{E}+10$ barns $)$ was exclusively used for nuclides with atomic number $\mathrm{Z}$ less than 7 , with the exception of B-11. Thermal scattering cross sections were produced for 6 additional bound nuclides which were processed at all the temperatures available in the JEFF-3.1 thermal scattering law data file [5]: $\mathrm{H}-1$ in light water, $\mathrm{H}-1$ in polyethylene, $\mathrm{H}-1$ in zirconium hydride (not contained in VITAMIN-B6), $\mathrm{H}-2$ in heavy water, $\mathrm{C}$ in graphite and $\mathrm{Be}$ in beryllium metal. VITJEFF31.BOLIB and MATJEFF31.BOLIB adopt the same problem-independent group structure of the VITAMIN-B6 library with 199 neutron and 42 photon energy groups, able to treat not only "fast" but also "thermal" neutron (water or graphite-moderated) reactor systems, through the adequate group structure at lower energies. It is underlined that the thermal neutron energy range below $5.043 \mathrm{eV}$, i.e., the range of the groups which include up-scatter, contains 36 groups. The photon energy group structure includes the top energy group with uppermost limit extended to $30 \mathrm{MeV}$. The neutron and photon weighting functions are the same adopted in VITAMIN-B6. The neutron weighting function is of the form typically chosen for fission reactor shielding problems, i.e., it consists of a smoothly varying combination of:

a Maxwellian thermal spectrum $(\mathrm{kT}=0.025 \mathrm{eV})$ : $\mathrm{W}_{1}(\mathrm{E})=\mathrm{C}_{1} \mathrm{Ee}^{-\mathrm{E} / \mathrm{kT}}$ from $1.0 \mathrm{E}-5 \mathrm{eV}$ to $0.125 \mathrm{eV}$,

a "l/E" slowing down spectrum:

$\mathrm{W}_{2}(\mathrm{E})=\mathrm{C}_{2} / \mathrm{E}$ from $0.125 \mathrm{eV}$ to $820.8 \mathrm{keV}$

and fission spectrum $(\theta=1.273 \mathrm{MeV})$ :

$\mathrm{W}_{3}(\mathrm{E})=\mathrm{C}_{3} \mathrm{E}^{1 / 2} \mathrm{e}^{\mathrm{E} / \theta}$ from $820.8 \mathrm{keV}$ to $20 \mathrm{MeV}$.

This corresponds to an IWT $=4$ option in GROUPR with the $\mathrm{C}_{1}, \mathrm{C}_{2}$ and $\mathrm{C}_{3}$ constants assuring the continuity of the spectrum function. The fission temperature $\theta$ has been adjusted to better reflect the neutron spectrum in a thermal reactor $(\theta=$ 1.273 MeV versus $\theta=1.41 \mathrm{MeV}$ for the library VITAMIN-E). The photon weighting spectrum consists of a $1 / \mathrm{E}$ spectrum with a "roll-off" of the spectrum at lower energies to represent photoelectric absorption and a similar drop-off of the spectrum at higher energies corresponding to the Q-value for neutron capture. This corresponds to the IWT $=3$ option in GAMINR. In both the libraries the order of scattering used for both neutrons and photons is $\mathrm{P} 7$, for nuclides with $\mathrm{Z}=1$ through $\mathrm{Z}=29$ (copper) and P5 for the remainder of the nuclides. The numerical values of the fractional error tolerances in NJOY for both the libraries, $0.1 \%$ for resolved resonance reconstruction and for linearization in RECONR and $0.1 \%$ for thinning in BROADR, were reduced with respect to the $0.2 \%$ corresponding values in VITAMIN-B6. About the available neutron fission spectra, the total (prompt + delayed) neutron 
Table 1. Summary of the k-effective calculated results for thermal and fast criticality safety benchmark experiments.

\begin{tabular}{|c|c|c|c|c|c|c|c|}
\hline \multicolumn{8}{|c|}{ Thermal Benchmark Results } \\
\hline & & & ENEA & VIT-B6 & VITJEF22 & VITJEFF31 & JEFF-3.1 \\
\hline ICSBEP & BNL-19302 & Experimental & Calculation & ORNL & ENEA & ENEA & OECD \\
\hline Handbook & (ENDF-202) & Results & Geometry/ & Report $^{\mathrm{a}}$ & Report $^{b}$ & Calculation & Report $^{c}$ \\
\hline & & keff $\pm \Delta$ keff & PN-SN & keff & keff & keff & $\operatorname{keff} \pm 1 \sigma(\mathrm{pcm})$ \\
\hline \multicolumn{8}{|c|}{ Unreflected Spheres of U-235 (as Uranyl Nitrate) in $\mathrm{H}_{2} \mathrm{O}$} \\
\hline- & ORNL-1 & $1.00026 \pm 0.0026$ & 1D Sph./ P3-S16 & 0.9965 & 0.99719 & 0.99690 & - \\
\hline - & ORNL-2 & $0.99975 \pm 0.0036$ & 1D Sph./ P3-S16 & 0.9964 & 0.99748 & 0.99712 & - \\
\hline - & ORNL-3 & $0.99994 \pm 0.0036$ & 1D Sph./ P3-S16 & 0.9935 & 0.99501 & 0.99463 & - \\
\hline- & ORNL-4 & $0.99924 \pm 0.0036$ & 1D Sph./ P3-S16 & 0.9950 & 0.99591 & 0.99549 & - \\
\hline- & ORNL-10 & $1.00031 \pm-$ & 1D Sph./ P3-S16 & 0.9961 & 0.99618 & 0.99805 & - \\
\hline \multicolumn{8}{|c|}{ Unreflected Spheres of Plutonium Nitrate in $\mathrm{H}_{2} \mathrm{O}$} \\
\hline - & PNL-1 & $1.0000 \pm-$ & 1D Sph./ P3-S16 & 1.0089 & 1.00898 & 1.00878 & - \\
\hline $\begin{array}{l}\text { PU-SOL-THERM-011 } \\
\text { (18.1) }\end{array}$ & PNL-3R & $1.0000 \pm 0.0052 *$ & 1D Sph./ P3-S16 & 0.9942 & 0.99558 \# & $0.99548 \#$ & - \\
\hline $\begin{array}{c}\text { PU-SOL-THERM-011 } \\
(18.6)\end{array}$ & PNL-4R & $1.0000 \pm 0.0052 *$ & 1D Sph./ P & 1.0013 & $1.00107 \#$ & $1.00106 \#$ & - \\
\hline $\begin{array}{l}\text { PU-SOL-THERM-011 } \\
(16.5)\end{array}$ & PNL-5R & $1.0000 \pm 0.0052 *$ & 1D Sph./ P3-S16 & 1.0065 & 1.00703 \# & $1.00678 \#$ & - \\
\hline \multicolumn{8}{|c|}{ Fast Benchmark Results } \\
\hline PU-MET-FAST-001 & JEZEBEL & $1.0000 \pm 0.002 *$ & 1D Sph./ P5-S16 & 0.9970 & $0.99598 \#$ & $0.99890 \#$ & $1.00010 \pm 19$ \\
\hline U233-MET-FAST-001 & JEZEBEL-23 & $1.0000 \pm 0.001 *$ & 1D Sph./ P5-S16 & 0.9934 & $0.96462 \#$ & $1.00383 \#$ & $1.00445 \pm 37$ \\
\hline PU-MET-FAST-002 & JEZEBEL-PU & $1.0000 \pm 0.002 *$ & 1D Sph./ P5-S16 & 0.9980 & $0.99781 \#$ & $1.00277 \#$ & $1.00428 \pm 67$ \\
\hline U233-MET-FAST-006 & FLATTOP-23 & $1.0000 \pm 0.0014^{*}$ & 1D Sph./ P5-S16 & 1.0032 & $0.97094 \#$ & $1.00670 \#$ & $1.00608 \pm 44$ \\
\hline PU-MET-FAST-006 & FLATTOP-PU & $1.0000 \pm 0.0030^{*}$ & 1D Sph./ P5-S16 & 1.0029 & $0.98928 \#$ & $1.00306 \#$ & $1.00267 \pm 43$ \\
\hline HEU-MET-FAST-001 & GODIVA & $1.0000 \pm 0.001^{*}$ & 1D Sph./ P5-S16 & 0.9960 & $0.99442 \#$ & $0.99550 \#$ & $0.99644 \pm 19$ \\
\hline HEU-MET-FAST-028 & FLATTOP-25 & $1.0000 \pm 0.0030^{*}$ & 1D Sph./ P5-S16 & 1.0018 & $0.99194 \#$ & $1.00235 \#$ & $1.00275 \pm 25$ \\
\hline- & ZPR-3/12 & $1.0000 \pm-$ & 2D RZ/ P3-S8 & 1.0109 & 1.00622 & $1.00546 \#$ & - \\
\hline - & ZPR-3/11 & $1.0000 \pm 0.0025$ & 2D RZ/ P3-S8 & 1.0141 & 1.00335 & 0.99790 & - \\
\hline $\begin{array}{l}\text { IEU-MET-FAST-007 } \\
\text { (simplified model) }\end{array}$ & BIG TEN & $1.0045 \pm 0.0070^{*}$ & 2D RZ/ P3-S8 & - & 8 \# & 0.99716 \# & $0.99777 \pm 27$ \\
\hline - & ZPR-6/7 & $1.0000 \pm 0.001$ & 2D RZ/ P3-S8 & 1.0150 & 0.99343 & 0.99724 & - \\
\hline- & ZPR-6/7 & $1.0000 \pm 0.001$ & 1D Sph./ P5-S16 & - & 0.99420 & 0.99716 & - \\
\hline
\end{tabular}

(*) Experimental data taken from the ICSBEP Handbook (September 2004 Edition) [13].

(\#) Geometrical-compositional model taken from the ICSBEP Handbook (September 2004 Edition) [13].

(a) ORNL-6795/R1, NUREG/CR-6214 Revision 1, p. 94 [1].

(b) ENEA Internal Report FIS-P815-001, April 16, 2003 [2].

(c) OECD/NEA Data Bank, JEFF Report 21, Appendix 2, 2006 [5].

fission spectra $\chi$ for U-235, U-238 and Pu-239, obtained from the specific GENDF files through the ENEA version of SMILER and the ICE modules of SCAMPI, were included in tabulated form into each package of the two libraries. It is underlined that the corresponding data in VITAMIN-B6 and VITJEF22.BOLIB/MATJEF22.BOLIB include only the prompt spectrum components.

\section{Examples of VITJEFF31.BOLIB validation}

VITJEFF31.BOLIB was tested on about 90 thermal, intermediate and fast spectrum neutron criticality benchmark experiments. A partial set of $\mathrm{k}_{\text {eff }}$ results for 9 thermal and 11 fast neutron benchmark experiments is presented in table 1 , where are compared the performances of the VITAMIN-B-6, VITJEF22.BOLIB and VITJEFF31.BOLIB libraries. The experimental details (geometry, composition and $\mathrm{k}_{\mathrm{eff}}$ ) were taken from refs. $[13,14]$. The principal intention was to prove that the results obtained were consistent with those reported in the OECD/NEADB JEFF-3.1 official report [5]. The calculations were performed using SCAMPI to produce the self-shielded working libraries, through the BONAMI module. The $\mathrm{k}_{\mathrm{eff}}$ results were calculated in spherical geometry through the XSDRNPM code (1D SN) [8] and in cylindrical geometry through the DORT-3.2 code (2D SN) [11]. Part of the $\mathrm{k}_{\mathrm{eff}}$ results obtained with VITJEFF31.BOLIB were compared with the MCNP-4C3/JEFF-3.1 Monte Carlo official validation results [5]. The $\mathrm{k}_{\mathrm{eff}}$ results obtained with VITJEFF31.BOLIB and VITJEF22.BOLIB for the thermal benchmarks are very near to those obtained by ORNL with VITAMIN-B6 [1]. The $\mathrm{k}_{\mathrm{eff}}$ result for ORNL-10, obtained with VITJEFF31.BOLIB, appears the closest to the corresponding experimental value while the $\mathrm{k}_{\mathrm{eff}}$ results for the fast benchmarks show that the deviations from the MCNP-4C3/JEFF-3.1 results [16] are very small. Moreover the $\mathrm{k}_{\mathrm{eff}}$ calculated results for the U-233 benchmark experiments (FLATTOP-23 and JEZEBEL-23), obtained through VITJEFF31.BOLIB, 
permit to appreciate an evident improvement with respect to the corresponding results of VITJEF22.BOLIB. The $\mathrm{k}_{\mathrm{eff}}$ results from VITJEF22.BOLIB, absolutely consistent with the MCNP/JEF-2.2 OECD/NEADB official results [15], are in fact strongly underestimated (more than $3000 \mathrm{pcm}$ ) while the corresponding VITJEFF31.BOLIB results are much less severely overestimated.

\section{Conclusion}

The present libraries will be distributed by the OECD/NEADB together with the ENEA-Bologna revised version of SCAMPI, which is able to read, differently from the presently distributed OECD/NEADB SCAMPI version, all NJOY-99 processed data files based on JEFF-3.1 or ENDF/B-VII.0. The generation of a collapsed working library of self-shielded cross sections in FIDO-ANISN format, dedicated to shielding and LWR pressure vessel nuclear safety transport analyses, was completed: the ENEA version of SCAMPI was successfully employed in the collapsing of VITJEFF31.BOLIB into the BUGLE-96 $(47 n+20 \gamma$ energy groups) group structure [1]. It is foreseen an effort to produce similar libraries based on ENDF/B-VII.0. It is considered important to extend further the availability of group-wise cross section libraries to offer, in particular, to the users of 3D deterministic codes like THREEDANT and TORT, a flexible possibility to generate working cross section libraries based on updated data files. Finally it is noted that the use of the deterministic codes is often preferred by the industrial organizations that must fulfil quality assurance procedures in reactor safety calculations. In fact there is no need, as in the case of the Monte Carlo codes, to justify the validity of the statistics adopted since the deterministic codes are based on rigorous analytical solutions of the neutral particle transport equations.
The authors want to thank Dr E. Sartori (OECD/NEA Data Bank) for his valuable support.

\section{References}

1. J.E. White et al., ORNL, NUREG/CR-6214, Revision 1, ORNL6795/R1, 1996, DLC-0184/ZZ VITAMIN-B6.

2. M. Pescarini et al., ENEA FIS-P815-001, April 16, 2003, NEA1699/01 ZZ VITJEF22.BOLIB.

3. M. Pescarini et al., ENEA FIS-P815-007, June 6, 2004, NEA-1740/01 ZZ MATJEF22.BOLIB.

4. R. Orsi, BOT3P Version 5.2, ENEA FPN-P9H6-002, March 8, 2007, NEA-1678/08 BOT3P5.2.

5. The JEFF-3.1 Nuclear Data Library, JEFF Report 21, OECD/NEA Data Bank, 2006.

6. R.E. MacFarlane, NJOY-99, "README0", PSR-0480/02, December 31, 1999.

7. V. Sinitsa, Activity Report of ENEA Research Fellowship, May 31, 2006 (private communication).

8. SCAMPI, ORNL, RSIC Peripheral Shielding Routines Collection, PSR-352, September 1995.

9. TRANSX 2.15, RSIC Peripheral Shielding Routines Collection, PSR-317, February 1995.

10. DANTSYS 3.0, ORNL, RSIC Computer Code Collection CCC547, August 1995.

11. DOORS3.1, ORNL, RSIC Computer Code Collection CCC-650, August 1996.

12. I.I. Bondarenko et al., Group Constants for Nuclear Reactors Calculations, (Consultants Bureau, New York, 1964).

13. ICSBEP, OECD/NEA NSC, NEA/NSC/DOC(95)03, September 2004 Edition.

14. Cross Section Evaluation Working Group Benchmark Specifications, BNL-19302 (ENDF-202), November 1974.

15. The JEF-2.2 Nuclear Data Library, JEFF Report 17, OECD/NEA Data Bank, April 2000.

16. S. Van der Marck (private communication). 